\title{
A novel interferometric liquid refractometer
}

\author{
D. J. Webb, ${ }^{\text {a) R.P. Tatam, }}{ }^{\text {b) }}$ and D. A. Jackson \\ Physics Laboratory, The University, Canterbury, Kent CT2 7NR, United Kingdom
}

(Received 3 March 1989; accepted for publication 21 June 1989)

We describe a novel form of liquid refractometer in which the opticai length of a cavity containing the liquid is monitored interferometrically.

The refractive index of liquids is conventionally measured using an Abbe refractometer or similar device. ${ }^{1}$ Recently, novel refractometers have been described which measure the attenuation of light guided by optical fibers within the liquid to be measured, ${ }^{2,3}$ and which monitor the angle of diffraction of light passing through a diffaction grating, the far side of which is exposed to the liquid under examination. ${ }^{4}$ We describe here a novel liquid refractometer based on interferometric principles.

The construction of the device is illustrated in Fig. 1. Light from a laser diode emitting at $815 \mathrm{~nm}$ with a nominal output power of $5 \mathrm{~mW}$ was launched into a length of single mode optical fiber and thereby transmitted to a buik-optic Michelson interferometer. On emerging from the fiber, the light was collimated, launched into the interferometer, and interference between light passing via each arm of the interferometer was observed at the detector. In one arm of the interferometer was placed a $10-\mathrm{cm}$ long cell to contain the fluid under examination. One of the mirrors was mounted on an Aerotech adjustable mount, while the second was fixed to a piezoelectric cylinder which was in turn mounted on a translation stage to permit adjustment of the optical path difference within the interferometer. The piezoelectric cylinder was driven with a simusoidal voltage at $2.9 \mathrm{kHz}$. The output from the photodetector was gated and band pass filtered at twice this frequency to produce an electronic carrier at a frequency of $5.8 \mathrm{kHz}$ phase modulated by the optical phase within the interferometer. This signal processing scheme has been previously described. ${ }^{5}$ The exact carrier frequency is unimportant so long as it is much higher than the frequency of the modulation applied to the laser diode.

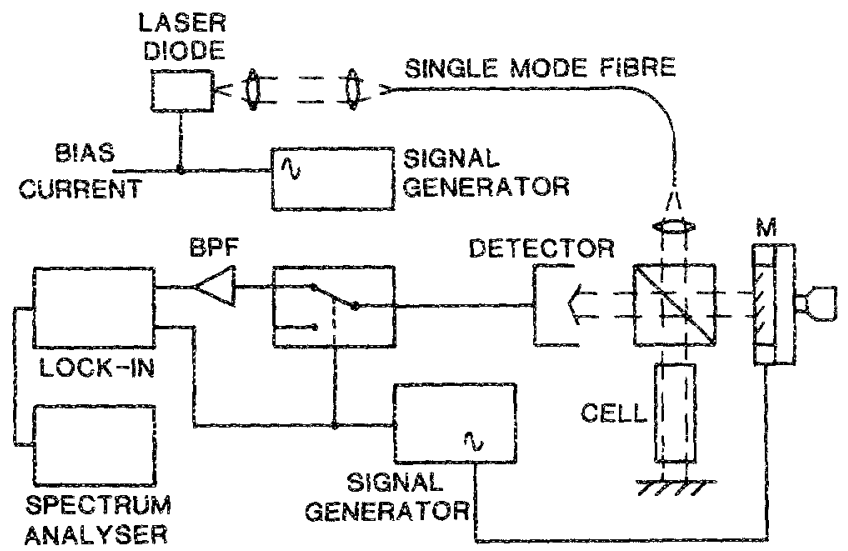

Fig. 1. Experimental arrangement. $\mathrm{BPF}=$ band pass filter. $\mathrm{M}=$ mirror mounted on piezoelectric disk attached to translation stage.
The electronic carrier was of the form $\cos (2 \pi f t+\phi)$, where $f$ is the carrier frequency $(5.8 \mathrm{kHz})$ and $\phi$ is the interferometric phase given by

$$
\phi=2 \pi n L v / c_{\text {, }}
$$

where $v$ is the emission frequency of the laser diode, $c$ is the velocity of light, and $n L$ is the optical path difference between the two arms of the interferometer. In the experiment, the laser frequency was sinusoidally modulated with a peak-to-peak frequency excursion of $200 \mathrm{MHz}$ and a modulation frequency of $10 \mathrm{~Hz}$ by varying the injection current. This resulted in a sinusoidal modulation of the optical phase, $\phi$, with an amplitude which from Eq. (1) may be seen to be proportional to the optical path difference within the interferometer, $n L$. This phase modulation was detected by comparing the carrier phase with that of the signal driving the piezoelectric cylinder using the lock-in amplifier as a phase meter. The signal of interest was the $10-\mathrm{Hz}$ component of the output from the lockin, the amplitude of which was proportional to the amplitude of the phase modulation within the interferometer. Other frequency components were present and, therefore, this signal was measured using the spectrum analyzer. An alternative method would have been to use a bandpass filter and an ac voltmeter.

With the cell initially containing air, the translation stage was adjusted to equalize the arms of the interferometer by minimizing the phase modulation. The cell was then filled in turn by a variety of liquids and the $10-\mathrm{Hz}$ component of the output of the lockin recorded. These results are tabulated in Table I, and displayed graphically in Fig. 2. Linear regression indicated a departure from linearity of $12.3 \mathrm{mV}$ corresponding to an error of 0.007 in the refractive index. This figure is open to question as the refractive indices were quoted at $589 \mathrm{~nm}$ and were in any case only accurate to 0.01 . Nevertheless, the results are promising, and demonstrate the feasibility of interferometric refractometry using this technique.

The conventional refractometers (Abbe, HilgerChance, etc.,...) are capable of much greater accuracies$10^{-5}$ may be obtained in a laboratory environment-but

TABLE 1. Experimental resuits.

\begin{tabular}{lcc}
\hline \hline Fluid & Signal level (mV rms) & Refractive index \\
\hline Air & 60 & 1.00 \\
Water & 654 & 1.33 \\
Isopropyl alcohol & 745 & 1.38 \\
Carbon tctrachloride & 900 & 1.46 \\
Benzene & 1000 & 1.50 \\
\hline
\end{tabular}




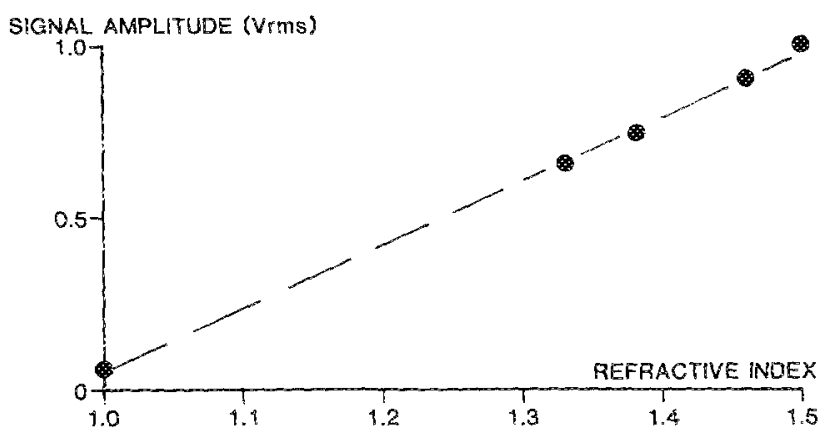

FIG. 2. Plot of refractive index against signal leveî,

such instruments do not readily lend themselves to automatic real time monitoring of refractive index. More recent instruments give accuracies of around $5 \times 10^{-3}$, with the advantages over conventional refractometers of simplicity ${ }^{4}$ or remote measurement. ${ }^{2}$ Work done in our laboratory on other applications of interferometry using a frequency modulated source suggests that the accuracy of the systen described here should be at least $10^{-3}$.

The main advantages of the scheme outlined in this note are first that, apart from the mirror oscillating with an amplitude of the order of the wavelength of light, there are no moving parts. Second, the refractive index of the liquid may be monitored in real time, thus permitting the scheme to be applied in the process control environment, Finally, the system is unaffected by variations in the absorption of the liquid under test, as this only affects the amplitude of the electronic carrier, and not the amplitude of the phase modulation imposed on that carrier. Further work is clearly needed to ascertain the fundamental performance limitations of the system.

a) Present address, and address of correspondence: University of Oxford, Department of Engineering Science, Parks Road, Oxford, OX1 3PJ, UK.

b) Present address: Applied Optics Group, School of Mechanical Engineering, Cranfeld Institute of Techuology, Cranfeld, BEDS, MK43 OAL, UK.

'R. S. Longhurst, Geometrical and Physical Optics, 3rd ed. (Longmans, London, 1962), p. 132.

${ }^{2}$ M. S. Meyer and G. L. Eesley, Rev. Sci. Instrum. 58, 2047 (1987).

${ }^{3}$ A. L. Harmer, in Froceedings of the First International Conference on Optical Fibre Sensors [IEE Conf. Publ. (London) 221, 104 (1983)].

${ }^{4}$ A. A. Zaidi, Rev. Sci. Instrum. 59, 1153 (1988).

${ }^{5}$ R. P. Tatam, J. D. C. Jones, and D. A. Jackson, Opt. Acta 33, 1519 (1986).

\title{
A trapezoidal function voltage generator for electrochemical applications
}

\author{
Rudolf Holze \\ Universität Oldenburg, Fachbereich Chemie, Canl-von-Ossietzky-Strasse 9-11, \\ D-2900 Oldenburg, West Germany \\ (Received 18 April 1989; accepted for publication 4 June 1989)
}

\begin{abstract}
An analog circuit based on standard operational amplifiers is described, which generates a trapezoidal voltage signal with rise and fall times as well as holding times variable over a wide range. Application of the circuit in spectroelectrochemistry is described, its properties are compared to currently available electrochemical instrumentation providing similar voltage functions.
\end{abstract}

The investigation of the electrode/electrolyte interface and interphase is possible with a rich variety of electrochemical, spectroelectrochemical, and other analytical methods. ${ }^{1-3}$ In most electrochemical methods the potential across this phase boundary is controlled by means of a potentiostat or a galvanostat. The potentiai can be modulated as a function of time with different potential (voltage) time functions in order to get information about the interface/interphase from the time dependent response measured as current-time or potential-time transients. Because of the special requirements a small number of commercially available function generators has been developed (for a recent review see Ref. 4). These instruments are in most cases based on simple analog circuitry and capable of only very limited potential-time programs; some microprocessor-controlled instruments are offered which provide a broader range of potential-time programs. Unfortunately, all these function generators are rather expensive. This is particularly troublesome when only a rather simple potential time function, e.g., a trapezoidal voltage, is required as a means to prepare electrodes for spectroelectrochemical investigations. In this case, the extreme precision and operational versatility of the commercial instruments characterized above would be unnecessary in most regards.

Such a trapezoidal voltage may include a voltage scan commencing at a negative (cathodic) value, stopping at a positive (anodic) limit for a certain time (up to a few seconds) and returning to its negative limit, where the sequence starts again immediately. It is very helpful in surface enhanced raman spectroscopy (SERS) with gold electrodes 